\title{
UNIQUENESS OF HAHN-BANACH EXTENSIONS AND UNIQUE BEST APPROXIMATION
}

BY

R. R. PHELPS

Introduction. The Hahn-Banach theorem states that a linear functional defined on a subspace $M$ of a normed linear space $E$ has at least one extension (with the same norm) to the whole of $E$. We intend to investigate those subspaces $M$ for which this extension is unique, that is, those subspaces $M$ having property $U$ : Each linear functional on $M$ has a unique norm-preserving extension to E. A. E. Taylor [14] and S. R. Foguel [4] have, between them, characterized those spaces $E$ for which every subspace has property $U$-they are precisely those $E$ having strictly convex conjugate. Another property which has long been of interest is that of unique best approximation, which we will call the Haar property: For each $x \in E$ there exists a unique $y \in M$ such that $\|x-y\|$ $=d(x, M)(=\inf \{\|x-z\|: z \in M\})$. There is an interesting and basic duality between these properties: The subspace $M$ has property $U$ if and only if its annihilator $M^{\perp}$ in $E^{*}$ has the Haar property. (This duality is not complete; we give examples showing that in the above statement the words "property $U$ " and "the Haar property" can not, in general, be interchanged.) The Taylor-Foguel theorem above is an immediate consequence of this theorem and of the fact that strictly convex spaces are precisely those in which every line through the origin has the Haar property.

By considering the dimension of certain extremal convex subsets of the unit sphere in $E^{*}$ [in $E$ ] we obtain a necessary condition that a subspace of finite dimension [deficiency] have property $U$ [the Haar property]. In the case of property $U$ this theorem is "sharp," in the sense that for certain special spaces, the condition expressed in the theorem characterizes property $U$ for finite dimensional subspaces. In the case of the Haar property this theorem enables us to conclude, for instance, that the spaces $c_{0}$ and $L_{1}[0,1]$ admit no Haar subspaces (i.e., subspaces having the Haar property) of finite deficiency. The latter complements the result that $L_{1}[0,1]$ admits no Haar subspace of finite dimension.

We obtain a theorem, related to one in [12], that gives a sufficient condition for a finite dimensional subspace to have the Haar property. In the case that the space $E$ is a closed ideal in $C(T)$ ( $T$ compact Hausdorff), this condition is also necessary. A corollary of the latter result establishes an extension of the classical Haar theorem [5] to $C_{0}(T)$ ( $T$ locally compact Hausdorff).

Presented to the Society, 1959; received by the editors May 14:, 1959. 
This paper is divided into three main sections. The first section contains theorems about general normed spaces. The second and third sections are devoted to a systematic application of these theorems to spaces of integrable functions and spaces of continuous functions, respectively. Most of the results from these two sections are summarized in a table at the end of the paper. A fourth and final section contains some remarks on applications and an unsolved problem, as well as an interesting theorem connecting reflexivity and best approximation, the proof of which is due to R. C. James.

The notion of extreme point (and the Krein-Milman theorem) plays an important role throughout this paper. In particular, the applications of the general theorems to special spaces $E$ make considerable use of known characterizarions of the extreme points of the unit cell of $E^{*}$.

1. Property $U$ and the Haar property in normed spaces. Throughout the paper $A \sim B$ will denote the set theoretic difference of the sets $A$ and $B$; the minus sign will be reserved for vector subtraction. We denote by $S$ the unit sphere $\{x:\|x\|=1\}$ in $E ; S^{*}$ denotes the corresponding subset of the conjugate space $E^{*}$. The symbol $\phi$ will denote the origin in $E$ and in $E^{*}$. If $M$ is a subspace of $E$ let $S_{M}=S \cap M$ and let $S_{M}^{*}=\left\{F \in M^{*}:\|F\|_{M}=1\right\}$, where $\|F\|_{M}=\sup \left\{|F(x)|: x \in S_{M}\right\}$. Let $M^{\perp}$ denote $\left\{f \in E^{*}: f(x)=0\right.$ for all $x \in M\}$, the annihilator subspace of $M$ in $E^{*}$. In what follows, we refer the reader to [3] for the definition or proof of any term or theorem which is otherwise left unexplained.

Since a subspace has property $U$ if and only if its closure has the property, and since any Haar subspace is necessarily closed, we will henceforth restrict our attention to closed subspaces, and assume that the word "subspace" means proper closed linear subspace. We say that a subspace $M$ of $E$ is proximinal if to each $x \in E$ there exists at least one $y \in M$ such that $\|x-y\|$ $=d(x, M)$. Thus, Haar subspaces are proximinal. Using the $w^{*}$-compactness of the unit cell in $E^{*}$, it is not difficult to see that every $w^{*}$-closed subspace of $E^{*}$ is proximinal. Indeed, suppose that $M \subset E^{*}$ is a $w^{*}$-closed subspace and that $f \in E^{*} \sim M$. Letting $C_{n}=f+\left[d(f, M)+n^{-1}\right] U^{*}$ be the cell of radius $1 / n+d(f, M)$ centered at $f$, the nested sequence $\left\{C_{n} \cap M\right\}$ consists of $w^{*}$ compact non-empty sets, and hence there is a point $g$ common to all of them. Clearly $g \in M$ and $\|f-g\|=d(f, M)$. It should also be noted that a subspace $M$ of $E^{*}$ is $w^{*}$-closed if and only if it is the annihilator of a subspace of $E$ (namely, of $M_{\mathrm{L}}=\{x \in E: f(x)=0$ for all $f \in M\}$ ). We will make considerable use of the following, which is immediate from the definition: A proximinal subspace $M$ of $E$ fails to have the Haar property if and only if there exist points $x \in E$ and $y \in M \sim\{\phi\}$ such that $\|x\|=\|x-y\|$ and $\|x\| \leqq\|x-z\|$ for each $z \in M$; or, such that $\|x\|=\|x+\lambda y\|$ for $|\lambda| \leqq 1$ and $d(x, M)=\|x\|$. We can (and occasionally will) assume that $\|x\|=1$.

In what follows we will frequently abbreviate the phrase " $M$ fails to have property $U^{\prime}$ to " $M$ is non- $U$." 
Theorem 1.1. A subspace $M$ has property $U$ in $E$ if and only if its annihilator $M^{\perp}$ has the Haar property in $E^{*}$.

Proof. Note first that if $f \in E^{*}$ then $d\left(f, M^{\perp}\right)=\|f\|_{M}$. Indeed, if $g \in M^{\perp}$ then $\|f\|_{M}=\sup \left\{|(f-g)(x)|: x \in S_{M}\right\} \leqq\|f-g\|$, so $\|f\|_{M} \leqq d\left(f, M^{\perp}\right)$. On the other hand, by the Hahn-Banach theorem we can choose $h \in E^{*}$ such that $h=f$ on $M$ and $\|h\|=\|f\|_{M}$. Then $f-h \in M^{\perp}$ and $\|f\|_{M}=\|f-(f-h)\| \geqq d\left(f, M^{\perp}\right)$. Now, if $M$ is non- $U$, there exist $F \in S_{M}^{*}$ and distinct functionals $f$ and $g$ in $S^{*}$ which extend $F$. Then $\phi \neq f-g \in M^{\perp}$ and $\|f\|=1=\|f-(f-g)\| \geqq d\left(f, M^{\perp}\right)$ $=\|f\|_{M}=\|F\|_{M}=1$, so $M^{\perp}$ does not have the Haar property. Conversely, if $M^{\perp}$ is not a Haar subspace, then there exist $h \in S^{*}$ and $f \in M^{\perp} \sim\{\phi\}$ such that $d\left(h, M^{\perp}\right)=1=\|h-f\|$. Then $h$ and $h-f$ have the same restriction $F$ to $M$, and $\|F\|_{M}=\|h\|_{M}=d\left(h, M^{\perp}\right)=1$, so $M$ is non- $U$.

Since a $w^{*}$-closed subspace $M$ of $E^{*}$ is the annihilator of $M_{\perp}$, we can conclude that such an $M$ has the Haar property if and only if $M_{\perp}$ has property $U$.

A normed space $E$ is strictly convex if $x, y \in S$ and $x \neq y$ imply $\|\lambda x+(1-\lambda) y\|$ $<1$ for $0<\lambda<1$, i.e. $S$ contains no line segments. This is the same as saying that each line through the origin has the Haar property; indeed, if $S$ were to contain a segment $[x, y]$, then the line determined by $[0, y-x]$ (a translation of $[x, y]$ to the origin) will fail to have the Haar property, while any line through $\phi$ which is non-Haar can be translated so that its intersection with $S$ is a segment. Now, each line through $\phi$ in $E^{*}$ is the annihilator of some closed hyperplane through $\phi$ in $E$, and the annihilator of such a hyperplane is a line through $\phi$ in $E^{*}$. Hence we can use Theorem 1.1 to see that $E^{*}$ is strictly convex if and only if each closed hyperplane through $\phi$ in $E$ has property $U$. Also, if $E^{*}$ is strictly convex, then every $w^{*}$-closed subspace of $E^{*}$ has the Haar property, and hence every subspace of $E$ has property $U$. These facts yield the Taylor-Foguel theorem of the introduction, as well as the following nonobvious result: Every subspace of $E$ has property $U$ if and only if every closed hyperplane through $\phi$ in $E$ has property $U$.

Can we interchange property $U$ and the Haar property in Theorem 1.1? Some light is shed on this question by the next two theorems.

Denote by $Q$ the natural isometry of $E$ into $E^{* *}$ defined by $(Q x)(f)=f(x)$ for $x \in E, f \in E^{*}$. We say that $E$ is reflexive if $Q(E)=E^{* *}$; this is equivalent (see $\$ 4$ ) to saying that every subspace in $E$ (or in $E^{*}$ ) is proximinal.

THEOREM 1.2. If $E$ is a reflexive Banach space, then a subspace $M$ of $E$ has the Haar property if and only if $M^{\perp}$ has property $U$.

The proof follows from Theorem 1.1 (applied to $E^{*}$ ), and the fact that $\left(M^{\perp}\right)^{\perp}=Q(M)$ in reflexive spaces.

THEOREM 1.3. If $M$ is a proximinal subspace of $E$ whose annihilator $M^{\perp}$ has property $U$, then $M$ is a Haar subspace. 
Proof. If $M$ fails to have the Haar property there exist points $x \in S$ and $y \in M \sim \phi$ such that $\|x-y\|=1=d(x, M)$. By the Hahn-Banach theorem there exists $f \in M^{\perp} \cap S^{*}$ such that $f(x)=1$, so $\|Q x\|_{M^{\perp}=1 \text {. Thus, the (common) }}$ restriction of $Q x$ and $Q(x-y)$ to $M^{\perp}$ has norm one, which shows that $M^{\perp}$ is non- $U$.

As we will see at the end of $\S 3$, the fact that $M^{\perp}$ has property $U$ does not imply that $M$ is proximinal, hence we cannot omit that hypothesis from the above theorem. Of more importance is the fact $(\S 3)$ that there exists a space $E$ containing Haar subspaces $M$ for which $M^{\perp}$ is non- $U$, so the converse to the above theorem is false.

If $A \subset E$, the span of $A$ is the (linear) set of all finite linear combinations of elements of $A$. The dimension $(\operatorname{dim} C)$ of a convex set $C$ is the dimension of the span of $C-x$, for any $x \in C$. The deficiency (def $M$ ) of a subspace $M$ is the dimension of the factor space $E / M$. If $x \in E \sim\{\phi\}$, let $A(x)$ $=\left\{f \in S^{*}: f(x)=\|x\|\right\}$. If $f \in E^{*} \sim\{\phi\}$, let $B(f)=\{x \in S: f(x)=\|f\|\}$. By the Hahn-Banach theorem, $A(x)$ is always nonempty, although $B(f)$ is nonempty if and only if the hyperplane $f^{-1}(0)$ is proximinal. Note that $A(x)$ is a $w^{*}$-closed, convex subset of $S^{*}$, and $B(f)$ is a closed convex subset of $S$.

THEOREM 1.4. If $M$ is a subspace of finite deficiency $n$, and if there exists an $f \in S^{*}$ such that $f(M)=0$ and $\operatorname{dim} B(f) \geqq n$, then $M$ is not a Haar subspace.

Proof. Pick $x \in B(f)$; since the span of $B(f)-x$ contains an $n$-dimensional subspace $L$, the finite dimensional set $(B(f)-x) \cap L$ has non-empty interior relative to $L$, and we may assume that $\phi$ is one of its interior points. This implies that every element in $L$ is a positive multiple of some element in $B(f)-x$. Now, since $\operatorname{dim} L=\operatorname{def} M$, if $L$ were to miss $M \sim\{\phi\}$ we could conclude that $E=L+M$. But $f$ vanishes on $L$ since it vanishes on $B(f)-x$; by hypothesis it vanishes on $M$, and hence it must vanish on $E$, a contradiction. Thus, there must exist an element $y \neq \phi$ in $L \cap M$. By taking a sufficiently small positive multiple of $-y$, we can assume that $-y \subset(B(f)-x) \cap M$. Now every element of $B(f)$ has norm one, so we have $\|x-y\|=1=\|x\|$; furthermore, if $z \in M$ then $\|x-z\| \geqq f(x-z)=1$. This shows that $M$ does not have the Haar property.

The dual theorem to this is the following.

THEOREM 1.5. If $M$ is a subspace of finite dimension $n$, and if there exists an $x \in S \cap M$ such that $\operatorname{dim} A(x) \geqq n$, then $M$ is non- $U$.

Proof. The functional $X=Q x$ is in $S^{* *} \cap\left(M^{\perp}\right)^{\perp}$, and we have $A(x)=B(X)$. The deficiency of $M^{\perp}$ is $n$, hence the previous theorem, applied to $M^{\perp}$, shows that the latter is not a Haar subspace. By Theorem 1, then, $M$ is non- $U$.

Suppose $x \in M \sim\{\phi\}$ and that $F \in M^{*},\|F\|_{M}=1$, is such that $F(x)=\|x\|$. Then every norm-preserving extension of $F$ is in $A(x)$ and if $\operatorname{dim} A(x)=0$ 
(i.e. $A(x)$ consists of a single point) then $F$ clearly has only one such extension. This gives an obvious sufficient condition for a finite dimensional subspace to have property $U$ (since for every functional $F$ on such a subspace there exists an $x \in M \sim\{\phi\}$ such that $F(x)=\|F\| \cdot\|x\|)$ i.e. if $\operatorname{dim} A(x)=0$ for every $x \in M \sim\{\phi\}$, then $M$ has property $U$. If $M$ is one dimensional, this is also a necessary condition. A point $x \neq \phi$ such that the line $R x$ (all real multiples of $x$ ) has property $U$ is called a smooth point of $E$; if every point has this property, $E$ itself is called smooth. Thus, the notion of subspaces with property $U$ is a generalization of the notion of smooth point. By Theorem 1.1 , and the fact that the $w^{*}$-closed hyperplanes of $E^{*}$ are precisely the subspaces of the form $(R x)^{\perp}, x \in E \sim\{\phi\}$, we can conclude that $E$ is smooth if and only if each weak*-closed hyperplane in $E^{*}$ has the Haar property.

It is not difficult to see that the sufficient condition of Theorem 1.5 is not a necessary one for a finite dimensional subspace to be non- $U$. This follows, essentially, from the obvious fact that two subspaces may intersect even if the dimension of one is less than the deficiency of the other. It is somewhat surprising, then, that the above condition is a necessary one in case $M$ is a finite dimensional subspace of $l_{1}$ or of $c_{0}$ (see $\S \S 2$ and 3).

Suppose that $C$ and $D$ are subsets of $E$, with $C \subset D$. We say that $C$ is an extremal subset of $D$ if (i) $C$ is convex and (ii) whenever $x$ and $y$ are in the convex hull of $D$ and the open segment ] $x, y$ [ meets $C$, then the closed segment $[x, y]$ is contained in $C$. If $C$ is an extremal subset of $D$, then any extremal subset of $C$ is also an extremal subset of $D$. Note that if $x \neq \phi,[f \neq \phi]$ then $A(x)[B(f)]$ is an extremal subset of $S^{*}[S]$. An extreme point of a set $D$ is an extremal subset of $D$ consisting of precisely one point. The set of all extreme points of $D$ is denoted by ext $D$. The following lemma, which gives a connection between the dimension of $A(x)$ and the number of its extreme points, will enable us to make use of known descriptions of ext $S^{*}$ for special spaces when applying Theorem 1.5 to such spaces.

LEMMA 1.6. If $x \neq \phi$ then $\operatorname{dim} A(x) \geqq n$ if and only if $A(x)$ contains an $(n+1)$-membered linearly independent subset of ext $S^{*}$.

Proof. The sufficiency portion is obvious. Suppose that $\operatorname{dim} A(x) \geqq n$. It will suffice to find $n+1$ points of ext $A(x)$ which form a linearly independent set, since $A(x)$ is extremal in $S^{*}$. Now $A(x)$ is a $w^{*}$-closed convex subset of the $w^{*}$-compact set $S^{*}$, hence by the Krein-Milman theorem [3] it has at least one extreme point. Using an induction, suppose that $\left\{f_{i}\right\}_{i=1}^{k} C \operatorname{ext} A(x)$ is a linearly independent set and that $1 \leqq k<n+1$. Then the dimension of the span $L$ of $\left\{f_{i}-f_{1}\right\}_{i=2}^{k}$ is $k-1<n \leqq \operatorname{dim} A(x)$. Hence $\left[A(x)-f_{1}\right] \sim L$ is nonempty and we can find $y \in E$ such that $Q y \neq 0$ on $A(x)-f_{1}$ but $Q y=0$ on $L$. Since $A(x)-f_{1}$ is $w^{*}$-compact and convex, $Q y$ attains its supremum on this set in a nonempty, $w^{*}$-compact extremal subset. Again, by the KreinMilman theorem, this set has an extreme point $f$ which is an extreme point of 
$A(x)-f_{1}$ and is not in $L$. Setting $f_{k+1}=f+f_{1}$ we have $\left\{f_{i}\right\}_{i=1}^{k+1} \subset \operatorname{ext} A(x)$. We can show that this set is linearly independent by using the fact that $f(x)=0$ and that $f \notin L$, which completes the proof.

In the sequel we will make frequent use of the following elementary fact.

Lemma 1.7. If $M$ is a subspace of $E$ with $0<\operatorname{dim} M<\infty$, and if $N$ is a subspace of $E^{*}$ such that $\operatorname{dim} N \leqq \operatorname{dim} M$, then $N \cap M^{\perp} \neq \phi$ implies $M \cap N_{\perp} \neq \phi$.

The proof follows by picking suitable bases for $M$ and $N$ and observing that if a determinant is zero, then its transpose is zero.

Some of the ideas used in the following theorem have been used previously by other authors (e.g., Ptak [9] in his proof of Haar's theorem for $C(T)$, $T$ compact Hausdorff); in particular, this theorem is closely related to one by Ivan Singer [12] which gives a necessary and sufficient condition that a finite dimensional subspace of a normed space has the Haar property. (Our condition is not necessary in general-see end of \$2.) The justification for its inclusion here is that its form is such as to yield quite readily the "hard" part of the proof of our extension of Haar's theorem, and the fact that the portion of the proof which overlaps with Singer's proof is considerably shorter than the latter.

Theorem 1.8. Suppose that $M$ is an n-dimensional subspace which fails to have the Haar property. Then there exists $x \in M \sim\{\phi\}$ and a linearly independent set $\left\{f_{i}\right\}_{i=1}^{n}$ of extreme points of $S^{*}$ such that $f_{i}(x)=0, i=1,2, \cdots, n$.

Proof. Since $M$ is finite dimensional it is proximinal, and hence there exists $y \in M \sim\{\phi\}$ and $z \in S$ such that $d(z, M)=1=\|z+\lambda y\|$ for $|\lambda| \leqq 1$. Let $L=M \oplus R z$; then there exists $F \in S_{L}^{*}$ such that $F(z)=1$ and $F(M)=0$. Now $S_{L}^{*}$ is the convex hull of its extreme points, and hence we can write $F$ $=\sum_{i=1}^{\boldsymbol{k}} \lambda_{i} F_{i}$, where $\lambda_{i}>0, \sum \lambda_{i}=1$ and $\left\{F_{i}\right\}_{i=1}^{\boldsymbol{k}} C \operatorname{ext} S_{L}^{*}$. If $|\lambda| \leqq 1$ we have $1=F(z)=F(z+\lambda y)=\sum \lambda_{i} F_{i}(z+\lambda y) \leqq \sum \lambda_{i}\|z+\lambda y\|=\|z+\lambda y\|=1$, hence $F_{i}(z+\lambda y)=\|z+\lambda y\|=1$, for each $i$. But taking $\lambda=0$ shows that $F_{i}(z)=1$ hence $F_{i}(y)=0$, for each $i$. Thus, each $F_{i} \in(R y)^{\perp}$. Since the latter is an $n$ dimensional subspace of $L^{*}$ we can pick a maximal linearly independent subset of the $F_{i}$ 's, say $\left\{F_{i}\right\}_{i=1}^{m}$, where $m \leqq n$. Now the unit cell of $L^{*}$ is an $(n+1)$ dimensional compact, convex set, so if $m<n$ we can, by the same argument as was used in the proof of Lemma 1.6, find additional extreme points $F_{m+1}, \cdots, F_{n}$ of $S_{L}^{*}$ such that $\left\{F_{i}\right\}_{i=1}^{n}$ is a linearly independent set. Thus, $F$ is an element (which vanishes on $M$ ) of the $n$-dimensional space spanned by the $F_{i}$. By Lemma 1.7 there exists $x \in M \sim\{\phi\}$ such that $F_{i}(x)=0, i=1,2$, $\cdots, n$. Now, for each $i$, the set $C_{i}$ of all norm-preserving extensions of $F_{i}$ to $E$ is a $w^{*}$-closed convex subset of $S^{*}$, and hence has an extreme point $f_{i}$. It follows from the fact that $F_{i} \in \operatorname{ext} S_{L}^{*}$ that $C_{i}$ is an extremal subset of $S^{*}$, hence $f_{i} \in \operatorname{ext} S^{*}$. The set $\left\{f_{i}\right\}_{i=1}^{n}$ is also linearly independent and vanishes on $x$, which completes the proof. 
The existence of a dual to this theorem seems unlikely, since (in general) the set $S \subset E$ may have no extreme points.

2. Spaces of integrable functions. A measure space $(T, \Sigma, \mu)$ is a set $T$, a $\sigma$-field $\Sigma$ of subsets of $T$ and a non-negative countably additive real valued measure $\mu$ defined on $\Sigma$. We usually refer to the elements of $\Sigma$ as the measurable subsets of $T$. We will assume that $T$ is a countable union of pairwise disjoint members of $\Sigma$ of finite measure, i.e. $T$ is $\sigma$-finite. The space $L_{1}(T, \Sigma, \mu)$ (briefly, $L_{1}$ ) is the Banach space of all real valued measurable functions $x$ on $T$ such that $\|x\|=\int_{T}|x| d \mu<\infty$, where (as usual) we identify any functions which are equal a.e. The conjugate space $L_{1}^{*}$ of $L_{1}$ can be identified [3] with the space $L_{\infty}(T, \Sigma, \mu)$ of essentially bounded functions on $T$, with norm $\|y\|=\operatorname{essup}_{t \in T}|y(t)|$. The linear functional $f$ on $L_{1}$ corresponding to $y \in L_{\infty}$ is defined by $f(x)=\int_{T} x y d \mu$, for all $x \in L_{1}$.

Lemma 2.1. Suppose that $M$ is an n-dimensional subspace of $L_{1}(T, \Sigma, \mu)$ and that for some $x \in M \sim\{\phi\}$, the set of zeros of $x$ contains $n$ pairwise disjoint sets of positive measure. Then $M$ is non- $U$.

Proof. Assume that $\|x\|=1$ and that $x=0$ on a set $A$ which contains the pairwise disjoint sets $A_{1}, \cdots, A_{n}, \mu\left(A_{i}\right)>0$. The $n$ characteristic functions $y_{i}$ of $A_{i}$ are in $L_{\infty}$ and form a linearly independent set. Hence, setting $y=\operatorname{sgn} x$ on $T \sim A, y=0$ on $A$, we have $\int_{T} x\left(y_{i}+y\right) d \mu=\int_{T}|x| d \mu=1=\left\|y_{i}+y\right\|$ for each $i$. Thus, the functionals corresponding to $y_{i}+y$ are in $A(x)$ for each $i$, which shows that $\operatorname{dim} A(x) \geqq n$. By Theorem 1.5, then, $M$ is non- $U$.

Theorem 2.2. Suppose that $M$ is a finite dimensional subspace of $L_{1}(T, \Sigma, \mu)$. Then $M$ has property $U$ if the set of zeros of each $x \in M \sim\{\phi\}$ is of measure zero. If $(T, \Sigma, \mu)$ contains no atoms, then this condition is also necessary.

Proof. If $M$ is non- $U$ there exist $y_{1}$ and $y_{2}$ in $L_{\infty}$ such that $y_{1} \neq y_{2},\left\|y_{1}\right\|=1$ $=\left\|y_{2}\right\|$, but the functionals on $L_{1}$ corresponding to $y_{1}$ and $y_{2}$ have a common restriction $F$ to $M$, with $\|F\|_{M}=1$. Since $M$ is finite dimensional there exists an $x \in M,\|x\|=1$, such that $F(x)=1$. Hence $\int_{T} x y_{1} d \mu=\int_{T} x y_{2} d \mu=1=\int_{T}|x| d \mu$, and it is not difficult to see that $y_{1}=y_{2}=\operatorname{sgn} x$ a.e. where $x \neq 0$. Since $y_{1} \neq y_{2}$, they differ on a set of positive measure and hence $x$ vanishes on a set of positive measure. To prove the converse, suppose that $(T, \Sigma, \mu)$ contains no atoms and that there exists an $x \in M \sim\{\phi\}$ which vanishes on a set $A$ of positive measure. Since $A$ is not an atom there exists $A_{1} \subset A$ such that $0<\mu\left(A_{1}\right)$ $<\mu(A)$. Hence $\mu\left(A \sim A_{1}\right)>0$ and we can find $A_{2} \subset A \sim A_{1}$ such that $0<\mu\left(A_{2}\right)$ $<\mu\left(A \sim A_{1}\right)$. Continuing, by induction, we can obtain $n$ pairwise disjoint subsets of $A$ of positive measure, where $n=\operatorname{dim} M$. By the above lemma, $M$ must be non- $U$.

An $L_{1}$ space whose underlying measure space is far from nonatomic is $l_{1}$, the space of absolutely summable sequences. Here, $T$ is the set of positive integers, $\Sigma$ the set of all subsets of $T$, and $\mu$ the "counting" measure, whose 
value at a subset $A$ is the number of elements in $A$. In this case, the conjugate space $L_{\infty}$ is the space $m$ of bounded sequences $y=\left\{y_{i}\right\}$ with the correspondence given by $\sum x_{i} y_{i}$, for all $x \in l_{1}$.

Theorem 2.3. Suppose that $M$ is an n-dimensional subspace of $l_{1}$. Then the following three properties of $M$ are equivalent:

(i) $M$ has property $U$.

(ii) For each $x \in M \sim\{\phi\}, \operatorname{dim} A(x)<n$.

(iii) Each $x \in M \sim\{\phi\}$ has at most $n-1$ zeros.

Proof. The fact that (i) implies (ii) follows directly from Theorem 1.5. To see that (ii) implies (iii), let $x \in M \sim\{\phi\}$ and define $Z(x)$ to be the set of positive integers $i$ such that $x_{i}=0$. We will show that card $Z(x) \leqq \operatorname{dim} A(x)$. Indeed, it is fairly easy to see that $y \in A(x)$ (which we assume to be a subset of $m$ ) if and only if $y_{i}=\operatorname{sgn} x_{i}$ for $i \notin Z(x)$, while $\left|y_{i}\right| \leqq 1$ for $i \in Z(x)$. Hence, letting $\delta^{i}$ be the sequence which is 1 at $i$ and zero elsewhere, and defining $z \in m$ to be the sequence equal to $\operatorname{sgn} x_{i}$ for $i \notin Z(x)$ and equal to zero for $i \in Z(x)$, we have that each element of $A(x)$ is of the form $z+\sum_{i \in Z(x)} \lambda_{i} \delta^{i}$, where $\left|\lambda_{i}\right| \leqq 1$ for each $i \in Z(x)$. Since the elements $\delta^{i}, i \in Z(x)$, form a linearly independent subset of $A(x)-z$, the dimension of the latter (and hence $\operatorname{dim} A(x)$ ) is at least card $Z(x)$, which was to be shown. To prove that (iii) implies (i), suppose that $M$ is non- $U$, so that there exists an $F \in M^{*},\|F\|_{M}=1$, such that $F$ admits two different extensions to $l_{1}$ of norm one. Denote the corresponding members of $m$ by $y^{1}$ and $y^{2}$. Since $M$ is finite dimensional there exists an $x \in M,\|x\|=1$, such that $F(x)=1$; hence $y^{1}$ and $y^{2}$ are in $A(x)$. Now if card $Z(x) \geqq n$, our proof is complete. If card $Z(x)=k<n$, we can find $x^{\prime} \in M \sim\{\phi\}$ such that card $Z\left(x^{\prime}\right)=n$, as follows. Use the above description of the elements of $A(x)$ to see that, if $y=y^{1}-y^{2}$, then $y_{i}=0$ for $i \notin Z(x)$. Hence $y=\sum_{i \in Z(x)} \alpha_{i} \delta^{i}$, with not all the $\alpha_{i}$ equal to zero. Pick $n-k$ distinct positive integers $i_{1}, i_{2}, \cdots, i_{n-k}$ which are not in $Z(x)$; then by taking $\alpha_{i_{j}}=0$ we have $y=\sum_{i \in Z(x)} \alpha_{i} \delta^{i}+\sum_{j=1}^{n-k} \alpha_{i j} \delta^{i_{i}}$, i.e. $y$ is a nontrivial linear combination of $n \delta^{i}$ s, which span a subspace $N$ of dimension $n$. Since $y^{1}$ and $y^{2}$ are extensions of $F, y=0$ on $M$, so by Lemma 1.7 there exists $x^{\prime} \in M \sim\{\phi\}$ which vanishes on $N$ and hence on the $n \delta^{i}$ 's. But this means that $x_{i}^{\prime}=0$ for $n$ integers $i$, which completes the proof.

The characterization of Haar subspaces of an $L_{1}$ space is much more difficult. Indeed, we will prove below that if $(T, \Sigma, \mu)$ contains no atoms then $L_{1}(T, \Sigma, \mu)$ contains no finite dimensional Haar subspaces. By using the same arguments as in Theorem 3.4 and the fact that if $(T, \Sigma, \mu)$ contains no atoms then $S \subset L_{1}(T, \Sigma, \mu)$ contains no extreme points, we can conclude that if $(T, \Sigma, \mu)$ contains no atoms, then $L_{1}(T, \Sigma, \mu)$ contains no Haar subspace of finite deficiency. One might be tempted to conclude that $L_{1}$ contains no Haar subspaces-this is not true, however. Indeed, if $A \in \Sigma, \mu(T \sim A)>0<\mu(A)$ and $M=\{x: x=0$ on $A\}$, then $M$ is a Haar subspace of $L_{1}(T, \Sigma, \mu)$. For if $y \in L_{1}$, 
let $x=y$ on $T \sim A,=0$ on $A$. Then $x \in M$ and if $z \in M, z \neq x$, we have $\|y-x\|$ $=\int_{A}|y| d \mu<\int_{A}|y| d \mu+\int_{T \sim A}|x-z| d \mu=\|y-z\|$, which shows that $x$ is the unique point in $M$ such that $\|y-x\|=d(y, M)$.

Lemma 2.4. Suppose that $M$ is a finite dimensional subspace of $L_{1}(T, \Sigma, \mu)$ and that there exists an extreme point $f$ of $S^{*}$ which annihilates $M$. Then $M$ is not a Haar subspace.

Proof. Since $f$ is extreme and $f(M)=0$ there exists $y$ in $L_{\infty}$ such that $|y|=1$ a.e. and $\int_{T} y x d \mu=0$ for each $x$ in $M$. Let $x_{1}, \cdots, x_{n}$ be a basis for $M$ and let $z=y \sum_{i=1}^{n}\left|x_{i}\right|$. Then $\sum x_{i} \neq \phi$ and for any scalars $a_{1}, \cdots, a_{n}$ we have $\left\|z-\sum a_{i} x_{i}\right\|=\int\left|y^{2} \sum\right| x_{i}\left|-y \sum a_{i} x_{i}\right| d \mu \geqq \int\left(\sum\left|x_{i}\right|-y \sum a_{i} x_{i}\right) d \mu=\|z\|$. Furthermore, wherever $\sum\left|x_{i}\right|-\sum x_{i} \neq 0$ we have $\operatorname{sgn}\left(y \sum\left|x_{i}\right|-\sum x_{i}\right)=\operatorname{sgn} y$ $=y$, so $\left\|z-\sum x_{i}\right\|=\int y\left(y \sum\left|x_{i}\right|-\sum x_{i}\right) d \mu=\|z\|$. Thus, $M$ is not a Haar subspace.

By Theorem 1.8, the converse to this is true for one-dimensional $M$; the span of $(0,0,1,0, \cdots)$ and $(1,-1,0,0, \cdots)$ in $l_{1}$ shows that the converse may fail for two-dimensional $M$.

The following theorem has been proved in [1] for the case $L_{1}[0,1]$.

TheOREM 2.5. If $(T, \Sigma, \mu)$ contains no atoms, then $L_{1}(T, \Sigma, \mu)$ contains no finite dimensional Haar subspaces ${ }^{(1)}$.

Proof. Let $M$ be a finite dimensional subspace with basis $x_{1}, \cdots, x_{n}$. We will exhibit an extreme point of $S^{*}$ which annihilates $M$. Indeed, write $x_{i}=x_{i}^{+}-x_{i}^{-}$, where $x_{i}^{+}, x_{i}^{-}$are $\geqq 0$ a.e., and define, for each $B$ in $\Sigma, m_{i}^{+}(B)$ $=\int_{B} x_{i}^{+} d \mu$ (and similarly for $m_{i}^{-}$). The $m_{i}^{+}, m_{i}^{-}$are finite nonatomic measures on $(T, \Sigma)$. By a theorem of Liapounoff, the subset of $2 n$-space consisting of all $2 n$-tuples of the form $\left(m_{1}^{+}(B), m_{1}^{-}(B), \cdots, m_{n}^{+}(B), m_{n}^{-}(B)\right)(B$ in $\Sigma)$ is convex. Hence we can choose $B$ in $\Sigma$ so that $m_{\boldsymbol{i}}^{+}(B)=m_{\boldsymbol{i}}^{+}(T) / 2, m_{\boldsymbol{i}}^{-}(B)$ $=m_{\boldsymbol{i}}-(T) / 2$. Defining $y=1$ on $B,-1$ on $T \sim B$, we have $\int y x_{i} d \mu=0$ for each $i$, so that the extreme point of $S^{*}$ determined by $y$ has the desired property.

3. Spaces of continuous functions. In this section we will consider spaces $E$ which are subspaces (not necessarily proper) of $C(T)$, the space of all real valued continuous functions on the compact Hausdorff space $T$, with the sup norm. We identify the conjugate space of $C(T)$ with the space rca $(T)$ [3] of all regular countably additive real valued set functions $\mu$ defined on the $\sigma$-field of all Borel subsets of $T$. Each such $\mu$ admits a decomposition $\mu=\mu^{+}$ $-\mu^{-}$, where $\mu^{+}$and $\mu^{-}$are non-negative members of rca $(T)$. The norm of $\mu$ is given by its total variation (so $\|\mu\|=\mu^{+}(T)+\mu^{-}(T)$ ) and the correspondence between $f \in C(T)^{*}$ and $\mu$ is given by $f(x)=\int_{T} x d \mu$, for all $x \in C(T)$.

(1) This theorem and its proof are due to the referee, who also showed that Lemma 2.4 could be strengthened to its present form. The theorem of Liapounoff used in the proof appears in Izv. Akad. Nauk. SSSR. vol. 4 (1940) pp. 465-478, MR 2. See also P. R. Halmos, The range of a vector measure, Bull. Amer. Math. Soc. vol. 54 (1948) pp. 416-421. 
Note that under pointwise multiplication $(x \cdot y(t)=x(t) \cdot y(t)$ for all $t \in T)$, $C(T)$ is an algebra. In any algebra $B$ an ideal $I$ is a linear subspace having the property that $x \in I$ and $y \in B$ implies $x y \in I$. It is shown in [7] that $I$ is a closed ideal in $C(T)$ if and only if $I=I_{A}$, the set of all continuous functions vanishing on the closed subset $A$ of $T$. The following lemma, which is of independent interest, will be of use in later theorems.

Lemma 3.1. If $A$ is a closed subset of the compact Hausdorff space $T$, then the ideal $I_{A}$ has property $U$ with respect to $C(T)$.

Proof. Suppose $F \in S_{I_{A}}^{*}$ and let $f$ be an extension of $F$ to $C(T)$ of norm one. Then there exists a unique $\mu \in \mathrm{rca}(T)$ corresponding to $f$, with $\mu^{+}(T)+\mu^{-}(T)$ $=1$. We show first that $\mu=0$ on the Borel subsets of $A$; indeed, if $\mu(B) \neq 0$ for some such subset $B$, then one of $\mu^{+}(B), \mu^{-}(B)$ is positive, say the former. Then $\mu^{+}(A)>0$ and if $x \in I_{A}$ we have $F(x)=f(x)=\int_{T \sim A} x d \mu \leqq \int_{T \sim A} x d \mu^{+}$ $\leqq\|x\| \cdot \mu^{+}(T \sim A) \leqq\|x\|\left[\mu^{+}(T)-\mu^{+}(A)+\mu^{-}(T)\right]=\|x\|\left[1-\mu^{+}(A)\right]$. But this implies that $\|F\|_{I_{\Lambda}<1}$, a contradiction. Suppose, now, that there exists another extension $g$ of $F$ and an $x \in C(T)$ such that $(f-g)(x)=\epsilon>0$. As above, the measure $\nu$ corresponding to $g$ vanishes on Borel subsets of $A$. Hence we can find an open neighborhood $U$ of the compact set $A$ such that $|\mu|(U \sim A)$ $<(\epsilon / 4)\|x\|$ and $|\nu|(U \sim A)<(\epsilon / 4)\|x\|$. (The measure $|\mu|$ is $\mu^{+}+\mu^{-}$.) Choosing $y \in C(T)$ such that $y=x$ on $T \sim U, y=0$ on $A$ and $\|y\| \leqq\|x\|$ we have $|f(y-x)|=\left|\int_{U \sim A}(y-x) d \mu\right| \leqq 2|| x \| \cdot|\mu|(U \sim A)<\epsilon / 2$. Similarly, $|g(y-x)|$ $<\epsilon / 2$. Since $y \in I_{A}$ we have $f(y)=g(y)$ and hence $|(f-g)(x)|<\epsilon$, a contradiction which completes the proof.

If $T$ is a compact Hausdorff space, and if the functional $f \in S^{*} \subset C(T)^{*}$ is an extreme point of $S^{*}$ then there exists a point $t \in T$ such that $f(x)=\epsilon x(t)$ for all $x \in C(T)$, where $\epsilon=1$ or -1 [3]. Conversely, for each $t \in T$, the functionals corresponding in this way to $t$ are in ext $S^{*}$. We now apply Theorem 1.5 and Lemma 1.6 to the case $E=C(T)$.

Theorem 3.2. Suppose that $M$ is an n-dimensional subspace of $C(T), T$ compact Hausdorf. Then if $M$ has property $U$, each $x \in M \sim\{\phi\}$ has the property that $|x(t)|=\|x\|$ for at most $n$ points $t \in T$. This latter condition is equivalent to saying that $\operatorname{dim} A(x)<n$. If $M$ is one-dimensional, this characterizes property $U$.

Proof. If $M$ has property $U$ then for each $x \in M \sim\{\phi\}, \operatorname{dim} A(x)<n$, by Theorem 1.5. By Lemma 1.6, $\operatorname{dim} A(x) \geqq n$ if and only if $A(x)$ contains an $(n+1)$-membered linearly independent subset of ext $S^{*}$. Using the characterization of ext $S^{*}$ mentioned above, we can see that this is equivalent to the existence of $n+1$ distinct points of $T$ at which $|x|$ attains its supremum. This, together with the remarks after Theorem 1.5 about one-dimensional subspaces, completes the proof.

If $T$ is a locally compact Hausdorff space let $C_{0}(T)$ be the space of con- 
tinuous functions on $T$ which vanish at infinity, i.e. those continuous $x$ having the property that for each $\epsilon>0$, the set $\{t:|x(t)| \geqq \epsilon\}$ is compact. Provided with the sup norm, $C_{0}(T)$ is a Banach space. If we compactify $T$ by adding a point $\infty$ at infinity, we obtain the compact Hausdorff space $T^{\prime}=T \cup\{\infty\}$. By an obvious imbedding, $C_{0}(T)$ can be considered as the subspace $I_{\{\infty\}}$ of $C\left(T^{\prime}\right)$. This remark justifies our consideration, whenever possible, of the spaces $I_{A}$. (By taking $A$ to be the empty set, we have $I_{A}=C(T)$.) If we take a countable set $T$ and topologize it by means of the discrete topology, $C_{0}(T)$ is isometric to $c_{0}$, the space of all sequences which converge to zero.

Theorem 3.3. If $M$ is an $n$-dimensional subspace of $c_{0}$, then $M$ has property $U$ if and only if for each $x \in M \sim\{\phi\}$ there exists at most $n$ integers $i$ such that $\left|x_{i}\right|=\|x\|$.

Proof. We consider $c_{0}^{*}$ to be the space $l_{1}$; it is easily verified that the extreme points of $S^{*}$ are the sequences $\delta^{i}(i=1,2, \cdots)$ which are 1 at $i, 0$ elsewhere. If $\|x\|=\left|x_{i}\right|$ then $\operatorname{sgn} x_{i} \delta^{i} \in A(x)$, so if $|x|$ attains its supremum at $n+1$ points $i_{1}, \cdots, i_{n+1}$, then $A(x)$ contains the $(n+1)$-membered linearly independent set $\operatorname{sgn} x_{i_{k}} \delta^{i_{k}}, k=1,2, \cdots, n+1$. By Lemma 1.6, $\operatorname{dim} A(x) \geqq n$ and by Theorem $1.5, M$ is non- $U$.

For any $x \in M$ let $J(x)$ be the set of all positive integers $i$ such that $\left|x_{i}\right|=\|x\|$. From the above, we have conv $\left\{\operatorname{sgn} x_{i} \delta^{i}: i \in J(x)\right\} \subset A(x)$. On the other hand, if the element $y \in l_{1}$ is in $A(x)$ we have $\|y\|=\sum\left|y_{i}\right|=1$, where $y=\sum y_{i} \delta^{i}$. If $y_{j} \neq 0$ for some $j \notin J(x)$ then (since $\left|x_{j}\right|<1$ ) we would have $1=\sum x_{i} y_{i} \leqq \sum\left|x_{i}\right|\left|y_{i}\right|<\sum\left|y_{i}\right|=1$, a contradiction. An equally simple argument shows that if $j \in J(x)$ and $y_{j} \neq 0$, then $\operatorname{sgn} y_{j}=\operatorname{sgn} x_{j}$. Hence $y=\sum_{i \in J(x)}\left|y_{i}\right| \operatorname{sgn} y_{i} \delta^{i}=\sum_{i \in J(x)}\left|y_{i}\right| \operatorname{sgn} x_{i} \delta^{i} \in \operatorname{conv}\left\{\operatorname{sgn} x_{i} \delta^{i}: i \in J(x)\right\}$, and hence the latter equals $A(x)$. Suppose now that $M$ is non- $U$, i.e. there exists $F \in S_{M}^{*}$ having two different extensions $z^{1}$ and $z^{2}$ to $c_{0},\left\|z^{1}\right\|=1=\left\|z^{2}\right\|$. (As above, $z^{1}$ and $z^{2}$ are considered to be elements of $l_{1}$.) Since $M$ is finite dimensional there exists $x \in M,\|x\|=1$, such that $F(x)=1$. Hence $z^{1}$ and $z^{2}$ are in $A(x)$. Let $k=$ card $J(x)$; if $k \leqq n$ we will find $y \in M,\|y\|=1$, such that $F(y)=1$ and card $J(y)>k$. By an obvious induction we can obtain an element $u \in M \sim\{\phi\}$ such that card $J(u)>n$, which will complete the proof. Since $z^{1}$ and $z^{2}$ are in $A(x)$, the element $z=z^{1}-z^{2}$ is a nontrivial linear combination of the elements $\delta^{i}, i \in J(x)$, and $z \in M^{\perp}$. Since $k \leqq n$, the subspace spanned by the $\delta^{i}$ is of dimension at most $n$, so, by Lemma 1.7 , there exists an element $v \in M \sim\{\phi\}$ vanishing on the $\delta^{i}$, i.e., $v_{1}=0$ for $i \in J(x)$. Multiplying by $\|v\|^{-1}$, we can assume that $\|v\|=1$. Since $v$ is a sequence converging to zero, there exists $j \notin J(x)$ such that $\|v\|=\left|v_{j}\right|$. Let $v^{\prime}=\operatorname{sgn} v_{j} \cdot v$ and let $w \in c_{0}$ be defined by $w_{i}=x_{i}$ if $i \in J(x), w_{i}=0$ otherwise. If $0<\alpha<1-\sup \left\{\left|x_{i}\right|: i \notin J(x)\right\}$ then $\left\|x-w+\alpha v^{\prime}\right\| \leqq 1$. On the other hand, if $\alpha$ is sufficiently large, $\left\|x-w+\alpha v^{\prime}\right\|>1$. Hence for some $\beta>0$ we have $\left\|x-w+\beta v^{\prime}\right\|=1$. Letting $y=x+\beta v^{\prime}$ we have 
$y \in M,\|y\|=1$, and since $\sum z_{i}^{1} v_{i}^{\prime}=0$ we have $F(y)=\sum z_{i}^{1} y_{i}=\sum z_{i}^{1} x_{i}=1$. Furthermore, $J(y) \supset J(x)$, and since $\|y\|=1$ we have $\left|y_{i}\right|=1$ for some $i \notin J(x)$. Hence card $J(y)>k=$ card $J(x)$, which was to be shown.

Unfortunately, the theorem analogous to the above is not true for every space of continuous functions. Indeed, if $c$ is the Banach space of convergent sequences (in the sup norm) we can obtain a two dimensional counter example as follows: Let $x_{1}=1, x_{n}=1-n^{-2}$ for $n>1$ and let $y_{1}=0, y_{n}=(-1)^{n} n^{-1}$ for $n>1$. It is not difficult to see that the subspace $M$ spanned by $x$ and $y$ satisfies the condition of the theorem, although the linear functional $F$ which is 1 at $x$ and 0 at $y$ has more than one norm preserving extension. Indeed, $F(x)$ $=1=\|F\|$, since $x_{n} \rightarrow 1$ implies $\|\alpha x+\beta y\| \geqq|\alpha|=|F(\alpha x+\beta y)|$ for all $\alpha, \beta$. Hence any sequence $\left\{z_{i}\right\}_{i=0}^{\infty}$ in $l_{1}$, considered as a functional on $c$ by the correspondence $(z, u)=\sum_{i=1}^{\infty} z_{i} u_{i}+z_{0} \lim u_{i}$, will be an extension of $F$ of norm one if we take $z_{0}$ and $z_{1} \geqq 0, z_{0}+z_{1}=1$ and $z_{i}=0$ for $i>1$.

We now consider Haar subspaces of $C(T)$. The results for finite dimensional subspaces are quite complete, but there seems to be no simple characterization of those of finite deficiency. We do have a characterization, however, for an interesting special case, which includes $C([0,1])$. A functional $f$ in $C(T)^{*}$ is non-negative if for each $x \in C(T)$ such that $x \geqq 0$ (i.e., $x(t) \geqq 0$ for all $t \in T)$ we have $f(x) \geqq 0$.

Theorem 3.4. Suppose that $T$ is a compact connected Hausdorff space and that $M$ is a proper subspace of finite deficiency in $C(T)$. Then $M$ is a Haar subspace if and only if $M=f^{-1}(0)$ (that is, $M$ is a subspace of deficiency 1) for some non-negative, nontrivial functional in $C(T)^{*}$.

Proof. If $M$ has the Haar property it is proximinal and there exists an $x \in S$ such that $\|x\|=1 \leqq\|x-y\|$ for each $y \in M$. Hence, by the Hahn-Banach theorem, there exists $f \in S^{*}$ such that $f(x)=1$ and $f(M)=0$. By Theorem 1.4, $\operatorname{dim} B(f)<n=\operatorname{def} M$, hence $B(f)$ is a compact extremal subset of $S$ and (by the Krein-Milman theorem) has extreme points which are necessarily extreme points of $S$. But, since $T$ is connected, the only extreme points of $S$ are the functions $\pm z$, where $z$ is identically 1 on $T$. Not both of these are in $B(f)$; assuming $z$ is, we have $B(f)=\{x\}=\{z\}$. Let $\mu \in$ rca $(T)$ correspond to $f$; then $1=f(z)=\int_{T} d \mu=\mu(T)=\|\mu\|$ and hence $\mu$ is non-negative; therefore $f$ is non-negative. Now if there were a point $y \in f^{-1}(0) \sim M$ we could assume (since $M$ is proximinal) that $\|y\|=1 \leqq\|y-u\|$ for each $u \in M$ and hence, as before, there would exist $g \in S^{*} \cap M^{\perp}$ such that $g(y)=1$. Still reasoning as above, we would have $y= \pm z$, a contradiction $(f(z) \neq 0=f(y))$.

To prove the converse, suppose $M=\{y: f(y)=0\}$, for some non-negative, nontrivial functional $f$ in $C(T)^{*}$. Then we have (by considering the measure corresponding to $f) f(-z)$ or $f(z)=\|f\|$; say the latter. For any $y \in M,\|f\|$ - $\|y-z\| \geqq f(y-z)=f(z)=\|f\|$, so $\|z\|=1 \leqq\|y-z\|$. If $M$ were not a Haar subspace we could find $y \in M \sim\{\phi\}$ such that $\|z \pm y\|=1$. Picking $t \in T$ such that 
$y(t)>0$ (or $-y(t)>0$ ) we would have $1<|z(t)+y(t)| \leqq\|z+y\|$ (or $1<\|z-y\|)$, a contradiction.

Using an argument as at the start of the above proof, together with the fact that the unit sphere in $c_{0}$ has no extreme points, we can see that the space $c_{0}$ contains no Haar subspaces of finite deficiency.

The next lemma gives an extension of Haar's theorem [5] to finite dimensional subspaces of a closed ideal $I_{A}$ in $C(T)$. Part of the necessity portion of the proof is a modification of Ptak's proof [9] for the case $I_{A}=C(T)$, while the sufficiency portion follows easily from Theorem 1.8.

Lemma 3.5. Suppose that $A$ is a closed subset of the compact Hausdorff space $T$, and that $M$ is an n-dimensional subspace of the closed ideal $I_{A}$. Then $M$ has the Haar property in $I_{A}$ if and only if each $x \in M \sim\{\phi\}$ has at most $n-1$ zeros in $T \sim A$.

Proof. If $M$ fails to have the Haar property then by Theorem 1.8 there exists a linearly independent subset $\left\{F_{i}\right\}_{i=1}^{n}$ of extreme points of the unit sphere of $I_{A}^{*}$ and an $x \in M \sim\{\phi\}$ such that $F_{i}(x)=0, i=1,2, \cdots, n$. By Lemma 3.1, each $F_{i}$ has a unique extension $f_{i}$ to $C(T)$ of norm one, and it is easily checked that each $f_{i}$ is an extreme point of the unit sphere of $C(T)^{*}$. Furthermore, the set $\left\{f_{i}\right\}_{i=1}^{n}$ is linearly independent. Hence, by the characterization of such extreme points mentioned previously, there exist distinct points $t_{1}, \cdots, t_{n}$ in $T$ such that $x\left(t_{i}\right)=0, i=1,2, \cdots, n$. If any $t_{i}$ were in $A$, we would have $\left.f_{i}\right|_{I_{A}}=F_{i}$ vanishing on $I_{A}$, a contradiction completing this portion of the proof. To prove the converse, suppose there exist $n$ distinct points $t_{1}, \cdots, t_{n}$ in $T \sim A$ and an $x \in M \sim\{\phi\}$ such that $\|x\|=1$ and $x\left(t_{i}\right)$ $=0, i=1, \cdots, n$. Letting $f_{i} \in C(T)^{*}$ be defined by $f_{i}(y)=y\left(t_{i}\right)$ for each $y \in C(T)$, the span $L$ of the $f_{i}, i=1, \cdots, n$ is a subspace of $C(T)^{*}$ of dimension at most $n$ which is contained in $(R x)^{\perp}$. By Lemma 1.7, then, there exists a nonzero linear combination $f=\sum_{i=1}^{n} \lambda_{i} f_{i}$ of the $f_{i}$ 's which vanishes on $M$; we may assume that $\sum\left|\lambda_{i}\right|=1$. Choose $y \in I_{A}$ such that $\|y\|=1$ and $y\left(t_{i}\right)$ $=\operatorname{sgn} \lambda_{i}$ if $\lambda_{i} \neq 0, i=1, \cdots, n$. Let $z=y(1-|x|)$; then $z \in I_{A}, z\left(t_{i}\right)=\operatorname{sgn} \lambda_{i}$ if $\lambda_{i} \neq 0,\|z\|=1$ and we have $\|z-x\| \leqq \sup \{|z(t)|+|x(t)|: t \in T\} \leqq 1$ since $|z|+|x|=|y| \cdot|1-| x||+|x| \leqq 1$. Now $f(z)=f(y)=1$, hence $1=f(z-w)$ $\leqq\|z-w\|$ for each $w \in M$ and therefore $\|z\|=1=\|z-x\|$. Thus, $M$ does not have the Haar property in $I_{A}$, which completes the proof.

THEOREM 3.6. If $T$ is a [locally] compact Hausdorff space and $M$ is an $n$-dimensional subspace of $C(T)$ [of $\left.C_{0}(T)\right]$ then $M$ has the Haar property if and only if each $x \in M \sim\{\phi\}$ has at most $n-1$ zeros.

The proofs follow immediately from the above lemma and the remarks preceding Theorem 3.3.

We consider again the space $L_{\infty}(T, \Sigma, \mu)$ defined in $\S 2$, without the assumption that $T$ is $\sigma$-finite. If the set $T$ is finite, this is simply the finite dimensional space $C(T)$ and Theorem 3.6 applies. In general, there exists a 
compact Hausdorff space $T_{1}$ which is extremally disconnected (that is, the closure of any open subset of $T_{1}$ is open) such that $L_{\infty}$ is isometric with $C\left(T_{1}\right)$ [3]. (Under pointwise multiplication, $L_{\infty}$ is an algebra, and this isometry preserves multiplication.) Thus, if $L_{\infty}$ contains a finite dimensional Haar subspace of dimension $>1$, then so does $C\left(T_{1}\right)$. But by a theorem of Sieklucki [13] (see §4), $T_{1}$ is then homeomorphic to a compact subset of the circle $K=\left\{(x, y): x^{2}+y^{2}=1\right\}$ in the Euclidean plane. It is not difficult to see that no infinite compact subset of $K$ can be extremally disconnected, hence we conclude that if the space $L_{\infty}(T, \Sigma, \mu)$ is infinite dimensional (so that $T_{1}$ is infinite), then it contains no finite dimensional Haar subspace of dimension $>1$. By duality, this implies that if the space $L_{1}(T, \Sigma, \mu)$ (with $T$ $\sigma$-finite) is infinite dimensional then it contains no subspace of finite deficiency greater than 1 which has property $U$. These remarks apply, of course, to the special spaces $m$ and $l_{1}$, respectively. What about one dimensional Haar subspaces of $L_{\infty}$ ? From Theorem 3.6 these are precisely the ones generated by those $x \neq \phi$ whose images (under the isometry with $C\left(T_{1}\right)$ mentioned above) have no zeros in $T_{1}$. This is equivalent to saying that no extreme point of $S^{*} \subset L_{\infty}^{*}$ vanishes at $x$. By [3, p. 443], the extreme points of $S^{*}$ are precisely the multiplicative linear functionals on $L_{\infty}$ or their negatives i.e., those nonzero functionals of the form $\pm f$, where $f(y z)=f(y) \cdot f(z)$ for all $y, z \in L_{\infty}$. Now such an $f$ takes on the value 1 at the element 1 (the function which equals 1 a.e.) and hence, if ess $\inf _{t \in T}|x(t)|>0$, we know that $x^{-1}=1 / x$ is essentially bounded (and therefore in $L_{\infty}$ ) and hence $1=f\left(x \cdot x^{-1}\right)=f(x) f\left(x^{-1}\right)$, so that $f(x) \neq 0$, i.e., if ess inf $|x|>0$, then no extreme point of $S^{*}$ vanishes at $x$. The converse follows from the lemma below, so we have $M=R x(x \neq \phi)$ is a onedimensional Haar subspace of $L_{\infty}(T, \Sigma, \mu)$ if and only if ess inf $|x|>0$. If $T$ is $\sigma$-finite, the duality Theorem 1.1 gives a characterization of $U$ subspaces of deficiency 1 in $L_{1}(T, \Sigma, \mu)$.

Lemma 3.7. If $x \in L_{\infty}(T, \Sigma, \mu)$ and ess inf $|x|=0$, then some multiplicative linear functional vanishes at $x$. If $(T, \Sigma, \mu)$ contains no atoms, then for each integer $m>0$ there exists an m-membered linearly independent set of such functionals vanishing at $x\left({ }^{2}\right)$.

Proof. The proof of the first assertion is obtained from the following proof by taking $m=1$ (and letting $\left.T_{m}=T\right)$. Assume, then, that $(T, \Sigma, \mu)$ contains no atoms. We show first that there exist $m$-pairwise disjoint sets $T_{j}, j=1,2$, $\cdots, m$, of positive measure such that ess $\inf _{t \in T_{j}}|x(t)|=0$ for each $j$. Indeed, if $x=0$ on a set of positive measure we can use the same argument as in the proof of Theorem 2.2 to obtain the $T_{j}$. If $|x|>0$ a.e. let

$$
T^{n}=\left\{t:(n+1)^{-1}<|x(t)| \leqq n^{-1}\right\} .
$$

(2) We wish to thank Professor Meyer Jerison for pointing out this lemma and suggesting its proof. He also points out that it is known that at least $2^{2 \times 0}$ such functionals exist. 
These sets are measurable, pairwise disjoint, and we can find an infinite subsequence, call it $\left\{T^{n}\right\}_{n=1}^{\infty}$, consisting of sets of positive measure. Letting $P_{k}$ be the $k$ th prime number, define $T_{j}=\bigcup_{n=1}^{\infty} T^{P^{n}}$ (so that $T_{j}$ is the union of the members of a subsequence of $\left\{T^{n}\right\}$ which has no terms in common with the subsequence associated with $T_{k}$ for $k \neq j$ ).

Now define $x_{j}$ to be equal to $x$ on $T_{j},=1$ elsewhere. Let $y_{j}=1$ on $T_{j},=x$ elsewhere; then $x=x_{j} y_{j}$, so for each $j, x$ is a member of any ideal which contains $x_{j}$. Since ess inf $\left|x_{j}\right|=0, x_{j}^{-1}$ is not essentially bounded, hence is not in $L_{\infty}$ and therefore the ideal $\left\{y x_{j}: y \in L_{\infty}\right\}$ is a proper closed ideal (it does not contain 1) which (by the usual maximality arguments) is contained in a maximal ideal $I_{j}$. As shown in [7], $I_{j}=f_{j}^{-1}(0)$ for some multiplicative linear functional $f_{j}$. The set $\left\{f_{j}\right\}_{j=1}^{m}$ is linearly independent, for if $f_{1}=\sum_{j=2}^{m} \alpha_{j} f_{j}$, say, we could use the fact that the ideals $I_{j}$ are distinct and maximal to choose $z_{j} \in I_{j} \sim I_{1}$ for $j=2,3, \cdots, m$. Letting $y=z_{2} z_{3} \cdots z_{m}$, we have $f_{1}(y) \neq 0$ but $f_{j}(y)=0$ for $j=2,3, \cdots, m$, which completes the proof.

We can now conclude that if $(T, \Sigma, \mu)$ contains no atoms, then $L_{\infty}(T, \Sigma, \mu)$ contains no finite dimensional subspace having property $U$. Indeed, if $M$ is a subspace of $L_{\infty}, m=\operatorname{dim} M<\infty$, pick any $x$ of norm one in $M$. Either ess sup $x$ $=1$ or ess $\sup (-x)=1$; supposing the former is true, say, set $y=1-x$. Then ess inf $|y|=0$ and by the lemma there exists a linearly independent set of multiplicative linear functionals $\left\{f_{j}\right\}_{j=1}^{m}$ which vanish at $y$. But $0=f_{j}(y)$ $=1-f_{j}(x)$ implies that $f_{j} \in A(x), j=1,2, \cdots, m$. Hence $\operatorname{dim} A(x) \geqq m$ $=\operatorname{dim} M$, so $M$ is non- $U$ by Theorem 1.5.

There exist infinite dimensional subspaces of $L_{\infty}$ having property $U$. Indeed, since the isometry of $L_{\infty}$ with $C\left(T_{1}\right)$ preserves multiplication, any closed ideal in $L_{\infty}$ can be considered as a closed ideal $I$ of $C\left(T_{1}\right)$. As mentioned previously, $I=I_{A}$ for some closed subset $A$ of $T_{1}$, so it follows from Lemma 3.1 that $I$ has property $U$. In particular, if $B$ is a measurable subset of $T$, then $I_{B} \subset L_{\infty}$ is a closed ideal and hence has property $U$.

We can now produce some examples related to Theorem 1.3.

ExAmple. If $M$ is a finite dimensional subspace of the Banach space $c_{0}$, then $M^{\perp}$ is non- $U$.

Note, first, that by considering $M$ as a subspace of $m=c_{0}^{* *}$, we have $M=M^{\perp \perp}$. If $\operatorname{dim} M>1$ then $\operatorname{dim} M^{\perp \perp}>1$ and it follows from the remarks preceding Lemma 3.7 that $M^{\perp \perp}$ cannot be a Haar subspace. If $\operatorname{dim} M=1$ then $M=R x=M^{\perp \perp}$ for $x \neq \phi$ in $c_{0}$. Now $\lim \left|x_{i}\right|=0$, so if $x$ is considered as an element of $m$, surely ess inf $|x|=0$ and therefore $R x=M^{\perp \perp}$ is not a Haar subspace. In either case, then, $M^{\perp \perp}$ does not have the Haar property and therefore (by Theorem 1.1) $M^{\perp}$ is non- $U$.

Using Theorem 3.6, it is easy to see that $c_{0}$ contains finite dimensional Haar subspaces $M$; by the above, $M^{\perp}$ is non- $U$.

EXAMPLE. There exists a Banach space $E$ and a subspace $M \subset E$ such that $M^{\perp}$ has property $U$, but $M$ is not proximinal. 
Let $E=c_{0}$, so that $E^{*}=l_{1}$. It is not difficult to verify that if $y \in l_{1} \sim\{\phi\}$, then $(R y)_{\perp}=\left\{x \in c_{0}: \sum x_{i} y_{i}=0\right\}$ is proximinal if and only if $y$ (as a functional on $c_{0}$ ) attains its supremum on $S \subset c_{0}$, and hence if and only if $y_{i}=0$ for all but finitely many $i$. Thus, if we pick $y$ such that $y_{i} \neq 0$ for all $i$, then (by Theorem 2.3) $R y$ has property $U$, but $(R y)_{\perp}$ is not proximinal. Since $(R y)_{\perp}$ $=R y$, this completes the proof.

4. Remarks. An interesting and important question related to Haar's theorem is the following: For what compact Hausdorff spaces $T$ does $C(T)$ admit Haar subspaces of dimension greater than one? (It follows from Theorem 3.6 that the one dimensional subspace generated by the function which is identically one is always a Haar subspace.) Haar [5] showed the existence of a space $T$ which admitted no nontrivial Haar subspaces, and in $1956 \mathrm{~J}$. C. Mairhuber [8] showed that (under the assumption that $T$ is a compact subset of Euclidean space $E^{k}$ ) if $C(T)$ admits a Haar subspace of dimension $n>1$, then $T$ is homeomorphic to a compact subset of the circumference of a circle. If $n$ is even, this is a proper subset. This theorem has since been proved, independently, by K. Sieklucki [13] and P. C. Curtis [2] under the assumption that $T$ is merely a compact Hausdorff space. In view of our characterization of Haar subspaces of $C_{0}(T)$ in Theorem 3.6, it would be interesting to know for what locally compact Hausdorff spaces $T$ there exist nontrivial Haar subspaces of $C_{0}(T)$.

If $C$ is a closed convex subset of $E$, we call $C$ proximinal if to each $x \in E$ there exists at least one $y \in C$ such that $\|x-y\|=d(x, C)$. It is well known that every closed convex subset of a reflexive Banach space is proximinal. (This is easily deduced, for instance, from the weak compactness of the unit sphere in such a space.) Less well known, however, is the truth of the converse: If every closed convex subset of a Banach space $E$ is proximinal, then $E$ is reflexive. The proof of this, which was kindly communicated to me by Professor R. C. James, goes as follows: If $E$ is a nonreflexive Banach space it must contain a separable nonreflexive subspace $M$. James [6] has shown that if a separable Banach space is nonreflexive, then there exists a linear functional on the space which does not attain its supremum on the unit sphere. Hence, there exists $f \in S_{M}^{*}$ such that $f^{-1}(1)$ misses $S \cap M$. Considered as a subset of $E, f^{-1}(1)$ is a closed convex set which is not proximinal (the origin has no nearest point in $f^{-1}(1)$ ), which completes the proof. Since $f^{-1}(1)$ is a translate of a subspace, this result may be formulated as follows: $A$ Banach space $E$ is reflexive if and only if each subspace of $E$ is proximinal.

If $M$ is a proximinal subspace of $E$ and $x \in E$ let

$$
N(x)=\{y \in M:\|x-y\|=d(x, M)\} .
$$

The nonempty set $N(x)$ is closed and convex, and it is clear that $M$ is a Haar subspace if and only if $\operatorname{dim} N(x)=0$ for each $x \in E$. G. Rubinstein [11] has generalized Haar's theorem in a different direction: Supposing that $M$ is an 
$n$-dimensional subspace of $C(T), T$ compact metric, and $0 \leqq k \leqq n-1$, he obtains a necessary and sufficient condition that $\operatorname{dim} N(x) \leqq k$, for each $x \in E$. (For $k=0$, his result gives Haar's theorem.) It is not too difficult to extend all our results of $\$ 1$ in this direction, getting estimates on the dimension of the set of Hahn-Banach extensions of a given functional (in the case of property $U$ ) in terms of $\operatorname{dim} A(x)$, for $x \in M \sim\{\phi\}$, and estimating $\operatorname{dim} N(x)$ (in the case of the Haar property) in terms of the number of extreme points of $S^{*}$ vanishing on some $y \in M \sim\{\phi\}$. The methods used in doing this are the same as our present ones and the theorems so obtained are not as simply stated, hence we have preferred to keep the present versions.

\begin{tabular}{|c|c|c|c|c|}
\hline \multirow{2}{*}{ Space } & \multicolumn{2}{|c|}{ Finite dimensional } & \multicolumn{2}{|c|}{ Finite deficiency } \\
\hline & Haar subspaces & $U$ subspaces & Haar subspaces & $U$ subspaces \\
\hline$C(T)$ & 3.6 & $\begin{array}{l}\text { For } \operatorname{dim} .=1 \text { see } \\
3.2\end{array}$ & $\begin{array}{l}\text { For connected } T \\
\text { see } 3.4\end{array}$ & \\
\hline$L_{1}$ & None, see 2.5 & 2.2 & $\begin{array}{l}\text { None, see remarks } \\
\text { following } 2.3\end{array}$ & $\begin{array}{l}\text { None of def. }>1 ; \\
\text { for def. }=1 \text { see re- } \\
\text { marks preceding } \\
3.7\end{array}$ \\
\hline$L_{\infty}$ & $\begin{array}{l}\text { None of } \operatorname{dim} .>1 ; \\
\text { for } \operatorname{dim} .=1 \text { see } \\
\text { remarks preceding } \\
3.7\end{array}$ & $\begin{array}{l}\text { None; see remarks } \\
\text { following } 3.7\end{array}$ & & $\begin{array}{l}\text { None, if weak*- } \\
\text { closed; see } 1.3 \text { and } \\
2.5\end{array}$ \\
\hline$C_{0}(T)$ & 3.6 & & & \\
\hline$c_{0}$ & 3.6 & 3.3 & $\begin{array}{l}\text { None; see remarks } \\
\text { following } 3.4\end{array}$ & \\
\hline$l_{1}$ & $\begin{array}{l}\text { For dim. }=1 \text { see } \\
2.4\end{array}$ & 2.3 & & Same as $L_{1}$ \\
\hline
\end{tabular}

In [10] W. W. Rogosinski has considered systems of $n$ linear equations

$$
\sum_{k=0}^{\infty} a_{i k} x_{k}=c_{i}
$$$$
(1 \leqq i \leqq n)
$$

in an infinity of unknowns $x_{k}$, where the $n$ (real or complex) sequences $\left\{a_{i k}\right\}_{k=0}^{\infty}$ are assumed to be linearly independent and the $c_{i}$ are real or complex numbers. Two cases of special interest are when (i) we assume that $\lim _{k \rightarrow \infty} a_{i k}$ $=0(1 \leqq i \leqq n)$ and consider solutions $\left\{x_{k}\right\}$ such that $\sum\left|x_{k}\right|<\infty$, and (ii) we assume $\sum_{k=0}^{\infty}\left|a_{i k}\right|<\infty(1 \leqq i \leqq n)$ and consider solutions $\left\{x_{k}\right\}$ such that $\sup \left|x_{k}\right|<\infty$. Rogosinski's attention is directed mainly to mininal solutions 
$\left\{x_{k}^{*}\right\}$, i.e. solutions which minimize $\sum\left|x_{k}\right|$ and sup $\left|x_{k}\right|$ respectively. It is shown that this is equivalent to finding all Hahn-Banach extensions of functionals (defined by (1)) on the subspace of $c_{0}$ (resp. $l_{1}$ ) spanned by the $\left\{a_{i k}\right\}_{k=0}^{\infty}$. In the case that the $a_{i k}$ and $c_{i}$ are all real, our theorems (3.3 and 2.3) give necessary and sufficient conditions for (1) to have a unique minimal solution.

In the table on the preceding page, $L_{1}$ and $L_{\infty}$ are assumed to be infinite dimensional spaces over a $\sigma$-finite measure space containing no atoms, while $C(T)$ and $C_{0}(T)$ are over a compact Hausdorff and a locally compact Hausdorff space, respectively. A number in a particular place means the property in question is characterized by the theorem having that number.

The subspace of all functions vanishing on a fixed measurable (or compact) subset of $T$ has the Haar property in $L_{1}$ and $l_{1}$, while it has property $U$ in $C(T), C_{0}(T), c_{0}, m$ and $L_{\infty}$.

\section{BIBLIOGRAPHY}

1. N. I. Ahiezer and M. G. Krein, On some problems in the theory of moments, (Russian) Kharkov, 1938.

2. P. C. Curtis, $N$-parameter families and best approximation, Pacific J. Math. vol. 9 (1959) pp. 1013-1027.

3. N. Dunford and J. T. Schwartz, Linear operators, New York, Interscience, 1958.

4. S. R. Foguel, On a theorem by A. E. Taylor, Proc. Amer. Math. Soc. vol. 9 (1958) p. 325.

5. A. Haar, Die Minkowskische Geometrie und die Annäherung an stetige Funktionen, Math. Ann. vol. 78 (1918) pp. 294-311.

6. R. C. James, Reflexivity and the supremum of linear functionals, Ann. of Math. vol. 66 (1957) pp. $159-169$. 1953.

7. L. H. Loomis, An introduction to abstract harmonic analysis, New York, Van Nostrand,

8. J. C. Mairhuber, On Haar's theorem concerning Chebyshev approximation problems having unique solutions, Proc. Amer. Math. Soc. vol. 7 (1956) pp. 609-615.

9. V. Ptak, A remark on approximation of continuous functions, Czechoslovak Math. J. (83) vol. 8 (1958) pp. 251-256.

10. W. W. Rogosinski, On finite systems of linear equations with an infinity of unknowns, Math. Z. vol. 63 (1955) pp. 97-108.

11. G. Rubinstein, On a method of investigation of convex sets, Dokl. Akad. Nauk SSSR. vol. 102 (1955) pp. 451-454 (Russian).

12. Ivan Singer, On uniqueness of the element of best approximation in arbitrary Banach spaces, (Rumanian, French summary) Stud. Cerc. Mat. vol. 8 (1957) pp. 235-244.

13. K. Sieklucki, Topological properties of sets admitting the Tschebycheff systems, Bull. Acad. Polon. Sci. Cl. III vol. 6 (1958) pp. 603-606. 547.

14. A. E. Taylor, The extension of linear functionals, Duke Math. J. vol. 5 (1939) pp. 538-

Institute for Advanced Study, Princeton, New Jersey 